\title{
Sequence Confirmation of Modified Oligonucleotides Using IRMPD in the External Ion Reservoir of an Electrospray Ionization Fourier Transform Ion Cyclotron Mass Spectrometer
}

\author{
Kristin A. Sannes-Lowery and Steven A. Hofstadler \\ Ibis Therapeutics, A Division of Isis Pharmaceuticals, Carlsbad, California, USA
}

\begin{abstract}
Modified oligonucleotides continue to play an important role as antisense compounds that inhibit the expression of genes associated with metabolic disorders, cancer, and infectious diseases. Because the majority of modifications render these molecules refractory to standard enzymatic sequencing techniques, alternative sequencing methods which are fast and reliable are needed. In this work we explore how sugar and backbone modifications affect fragmentation patterns observed from oligonucleotides which are fragmented by infrared multiple photon dissociation in the external reservoir of an electrospray ionization Fourier transform ion cyclotron mass spectrometer. The modifications influence which fragment types (i.e., $\mathrm{a}_{\mathrm{n}}-\mathrm{B}$ versus $c_{n}$ ) dominate and the ease with which the oligonucleotides are fragmented. General observations for confirming the sequence of oligonucleotides are described. (J Am Soc Mass Spectrom 2003, 14, 825-833) (c) 2003 American Society for Mass Spectrometry
\end{abstract}

A ntisense compounds are modified oligonucleotides that bind messenger RNA and inhibit the expression of genes associated with metabolic disorders, cancer, and infectious diseases. Modified oligonucleotides are necessary to increase the binding of the oligonucleotides to the messenger RNA as well as to minimize the in vivo enzymatic degradation of the oligonucleotides [1]. Typically, modifications are made to the phosphate backbone and to the $2^{\prime}$ position of the sugar. Fast and reliable methods are needed for sequencing these oligonucleotides since standard enzymatic techniques are not compatible with the majority of these modifications.

Mass spectrometry has developed into a powerful tool for studying macromolecules of biological importance, including nucleic acids. Soft ionization techniques such as electrospray ionization [2-4] and matrixassisted laser desorption/ionization [5, 6] allow the introduction of large intact biomolecules into the gas phase for mass spectrometric analysis. In addition to accurate molecular weight determination, mass spectrometry in combination with different fragmentation schemes provides information about sequence and/or structure of the biomolecules. Seminal work by McLaf-

Published online June 23, 2003

Address reprint requests to Dr. S. A. Hofstadler, Ibis Therapeutics, Division of Isis Pharmaceuticals, 2292 Faraday Avenue, Carlsbad, CA 92008. E-mail: Shofstadler@isisph.com ferty and coworkers demonstrated the ability to obtain partial sequence information for DNA oligonucleotides up to 108 nucleotides in length using tandem mass spectrometry techniques on a FTICR spectrometer [7]. By combining various fragmentation schemes, it was shown that complete sequence coverage could be obtained for a 50-mer DNA [7-9]. Other researchers demonstrated that sequence information and identification of modified bases could be obtained by combining fragmentation schemes with ion trap mass spectrometry [10] and triple quadrupole mass spectrometry [11-13].

Based on the small amounts of sample required and the unambiguous signatures generated, mass spectrometry is emerging as the preferred platform on which to interrogate and sequence modified oligonucleotides. In order to faithfully sequence oligonucleotides containing desperate chemical modifications, an understanding of how the modifications influence the fragmentation pattern is essential. One of the motivating factors behind this work was the desire to rapidly confirm the purported sequence of a modified oligonucleotide regardless of the chemical modifications employed in the synthesis. While a mass measurement of an intact oligonucleotide that is consistent with the expected sequence is generally adequate, there are many cases in which sequence verification is a prerequisite to animal toxicity studies or clinical trials involving human subjects. It is possible that an oligonucleotide could be 
Table 1. Sequence, sugar, and backbone of oligonucleotides used in this study

\begin{tabular}{llll}
\hline Oligonucleotide & \multicolumn{1}{c}{ Sequence } & Sugar & Backbone \\
\hline \hline Oligo 1 & TGA5GGATG55AG5TTGGG5T & 2'-deoxyribose & Phosphodiester \\
Oligo 2 & TGA5GGATG55AG5TTGGG5T & 2'-deoxyribose & Phosphorothioate \\
Oligo 3 & GCGTTTGCTCTTCTTCTTGCG & 2'-deoxyribose & Phosphorothioate \\
Oligo 4 & GTTCTCGCTGGTGAGTTTCA & 2'-deoxyribose & Phosphorothioate \\
Oligo 5 & T5TT55A5TGT5TTTT5A & 2'-deoxyribose & Phosphorothioate \\
Oligo 6 & GATG55AGAGAGGATG55 & 2'-deoxyribose & Phosphorothioate \\
Oligo 7 & GCCCAAGCTGGCATCCGTCA & 2'-deoxyribose & Phosphorothioate \\
Oligo 8 & GCCCAAGCTGGCATCCGTCA & Ribose & Phosphorothioate \\
Oligo 9 & GCCCAAGCTGGCATCCGTCT & 2'O-methoxyethyl ribose & Phosphorothioate \\
Oligo 10 & GATG55AGAGAGGATG55 & 2'-deoxyribose, 2'-O-methoxylethyl ribose & Phosphorothioate \\
\hline
\end{tabular}

Note: $5=5$ methyl C.

erroneously synthesized with the correct base composition but incorrect sequence. Although the focus of this work is sequence confirmation, the observations and conclusions derived herein should be equally applicable to the de novo sequence determination of modified oligonucleotides. In this work, infrared multiple photon dissociation in the external reservoir of an electrospray ionization Fourier transform ion cyclotron resonance mass spectrometer (InHex IRMPD ESI-FTICR MS) is used to investigate the influence of chemical modifications on the fragmentation patterns of oligonucleotides.

\section{Experimental}

\section{Chemicals}

Table 1 shows the sequences and modifications of oligonucleotides used in this study. All oligonucleotides were synthesized using standard phosphoramidate chemistry. All oligonucleotides were purified by ethanol precipitation as ammonium salts. $15 \mu \mathrm{M}$ solutions of each oligonucleotide were prepared in 50:50 isopropanol/water solution containing $0.1 \%$ tripropylamine.

\section{Instrumentation}

All experiments were performed on a Bruker Daltonics (Billerica, MA) Apex 70e Fourier transform ion cyclotron resonance mass spectrometer. The spectrometer is equipped with an Analytica (Branford, CT) electrospray source utilizing a grounded ESI emitter, a glass desolvation capillary with countercurrent drying gas, and an external ion reservoir. The external ion reservoir consists of a biased skimmer cone, an rf-only hexapole ion guide and a gate electrode at the low-pressure end of the hexapole ion guide. Ions are accumulated in the external ion reservoir (concurrent with IR irradiation for the IRMPD experiments) for $0.5-1 \mathrm{~s}$ and pulsed into the Infinity trapped ion cell for analysis by FTICR [14]. The oligonucleotides were analyzed in the negative mode with the potential applied to the capillary exit maintained at $-50 \mathrm{~V}$ to avoid nozzle-skimmer fragmentation. The skimmer cone voltage is held at $0 \mathrm{~V}$ and the gate electrode toggles between $-20 \mathrm{~V}$ during accumulation and $20 \mathrm{~V}$ during ion ejection. The ions are excited and detect from 440 to $5000 \mathrm{~m} / \mathrm{z}$ values. Thus, fragment ions with $m / z$ values $<440$ such as $\mathrm{a}_{2}-\mathrm{B}$ and $\mathrm{w}_{1}$ will not be observed under these experimental conditions. All aspect of the experiment including data acquisition, processing, and plotting were performed using Bruker XMASS version 5.0 software running on a dual processor Pentium PC.

\section{IRMPD}

A $25 \mathrm{~W}$ continuous wave $\mathrm{CO}_{2}$ laser (Synrad Inc., Mukilteo, WA) operating at $10.6 \mu \mathrm{m}$ was used for all IRMPD experiments. Using standard IR-compatible mirrors and kinematic mirror mounts, the unfocused 3 $\mathrm{mm}$ laser beam was aligned to traverse through the length of the instrument from the infinity cell to the hexapole ion guide and finally impinge upon the skimmer cone [14]. The laser was operated at an output range of $10-28 \mathrm{~W}$ as measured at the entrance to the mass spectrometer; no attempt was made to determine the actual laser power in the hexapole ion guide. The ions were irradiated in the hexapole ion guide during the ion accumulation event.

\section{Data Analysis}

Using a computer program written in house, the monoisotopic neutral mass and the monoisotopic peak of the 1- to 5- change states of all possible $a_{n}, a_{n}-B, b_{n}, c_{n}$ $\mathrm{w}_{\mathrm{n}}, \mathrm{x}_{\mathrm{n}}$, and $\mathrm{y}_{\mathrm{n}}$ fragment ions were generated from the expected oligonucleotide sequence. The InHex IRMPD mass spectrum of the oligonucleotide is deconvoluted using ICR-2LS [15] which generates a list containing the identified peak, charge state, absolute abundance (arbitrary units) and calculated monoisotopic neutral mass. The list of predicted fragment ions is compared to the monoisotopic neutral masses calculated from the experimentally observed peaks and as shown for Oligo 7 in Figure 1B, the deconvoluted spectrum is labeled with the appropriate fragment. Since some fragment ions will overlap in the raw mass spectrum as shown in the 


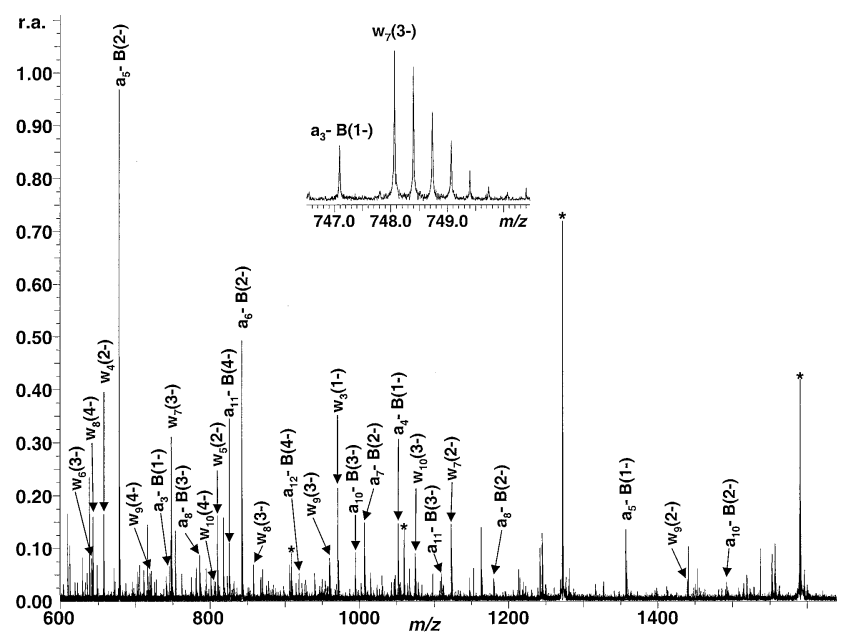

(a)

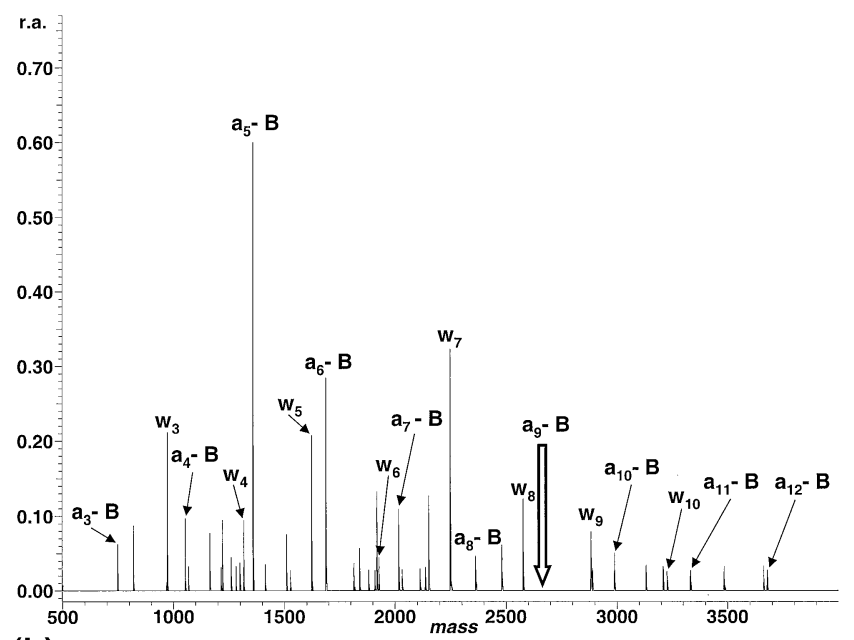

(b)

Figure 1. Mass spectrum of the InHex IRMPD fragmentation of the phosphorothioate deoxyoligonucleotide 5'-GCCCAAGCTGGCATCCGTCA-3': (a) raw mass spectrum and (b) deconvoluted mass spectrum. Only the $a_{n}-B$ and $w_{n}$ fragment ions have been labeled. In (a), peaks corresponding to residual intact oligonucleotide are labeled with an asterisk and the inset shows that closely space fragment ions can be resolved when ESI-FTICR-MS is used. In (b), the arrow marks the calculated mass of the $\mathrm{a}_{9}-\mathrm{T}$ fragment, which is clearly not observed.

inset of Figure 1a and may not be deconvoluted correctly, missing fragment ions necessary for sequence confirmation are searched for in the raw spectrum using the generated list of predicted fragments. If there are unidentified peaks with significant absolute abundance, it is verified that the peaks do not correspond to alternative sequences. When all fragment ions have been identified, the absolute abundance of each charge state for the individual fragment ions are summed to give a total absolute abundance of that fragment (i.e., the abundance of the 1- and 2- charge states of $a_{5}-A$ fragment are summed together to give a total absolute abundance for the $\mathrm{a}_{5}-\mathrm{A}$ fragment). The total fragmentation abundance of the oligonucleotide is then calculated by summing the total absolute abundance for each fragment and normalizing to 1 . This way, the abundance of the individual fragments represent a fraction of the total fragmentation abundance and can be displayed in bar graphs (e.g., Figure 2a). The observed order of preference for fragmentation on the $3^{\prime}$ side of a base for each fragment type was established by determining the percentage of fragmentation occurring $3^{\prime}$ to each base for a given fragment type, dividing the percentage of fragmentation at each base by the number of times that base occurs in the sequence and rank ordering based on the normalized percentage of fragmentation.

\section{Results and Discussion}

\section{Background}

Throughout this paper we use the standard oligonucleotide fragmentation nomenclature established by McLuckey et al. [16]. Several dissociation schemes have been used in combination with mass spectrometry to fragment DNA oligonucleotides of various lengths. These methods include nozzle-skimmer dissociation [17], collisional activated dissociation (CAD) [12, 13], infrared multiple photon dissociation (IRMPD) [18], blackbody infrared radiative dissociation (BIRD) [19], and multipole storage assisted dissociation (MSAD) [20]. Several groups have shown that modified bases influence fragmentation patterns of oligonucleotides and can be used to locate modified bases that occur naturally in damaged DNA [10, 21-24]. The effect of replacing the phosphate groups with methylphosphonates on the fragmentation patterns of DNA has also been investigated, indicating that fragmentation can occur at non-ionized linkage sites [25, 26].

Recently, we demonstrated the utility of performing IRMPD in an external ion reservoir [14]. In this scheme, a laser beam traverses the external ion reservoir during the ion accumulation event and causes fragmentation of the molecular ions. Because ions are exposed to a range of laser irradiation times, increased sequence coverage and fragmentation ion yields are observed. Additionally, because the external ion reservoir is at a relatively high pressure, the ions undergo a large number of low-energy collisions that stabilize metastable fragments, which would otherwise go undetected. Unlike in-cell CAD approaches that require transiently elevated analyzer cell pressure followed by an extended pump down time before excitation and detection, InHex IRMPD is performed without modulating the pressure in the analyzer cell and is thus very rapid. Owing to the inherent complexity of the resulting mass spectra, the FTICR is an ideal platform on which to make such measurements. For the spectra shown in this work, average resolving power in excess of 100,000 (FWHM) was routinely obtained concurrent with $<2 \mathrm{ppm}$ mass measurement error. Such performance facilitates the unambiguous assignment of fragments. 


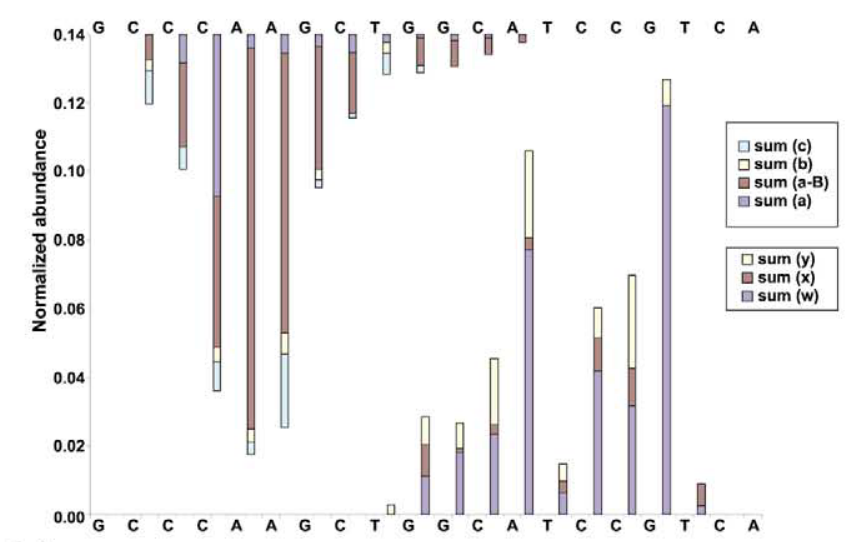

(a)

Oligo 8 (ribose) fragmentation pattern

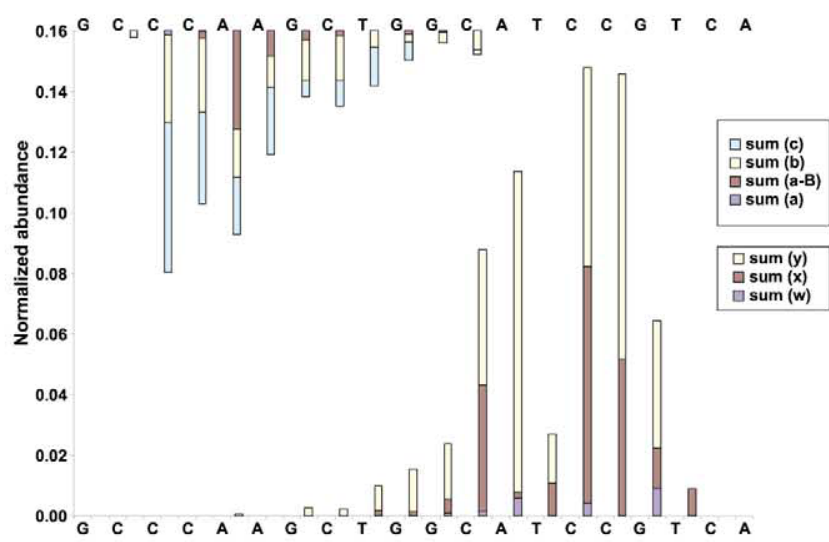

(b)

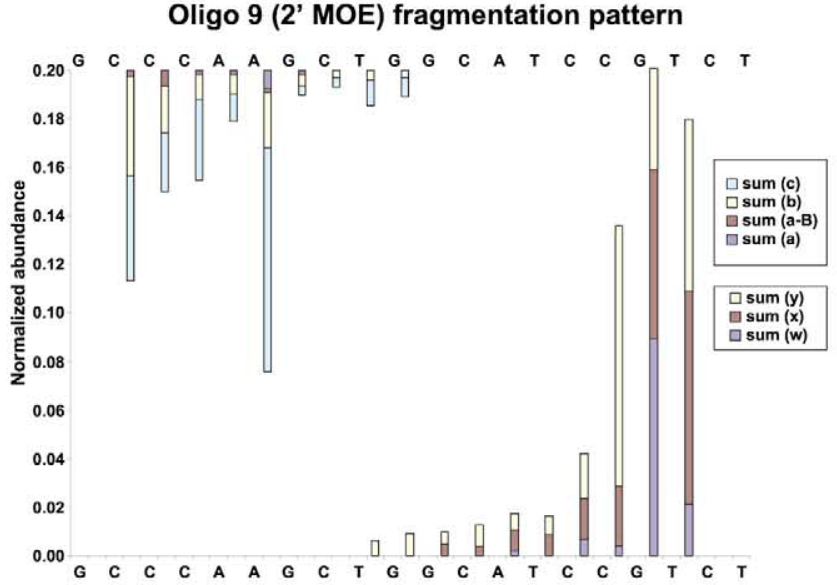

(c)

Figure 2. InHex IRMPD fragmentation patterns for the phosphorothioate oligonucleotide 5'GCCCAAGCTGGCATCCGTCA-3' containing 2' substitutions of (a) deoxyribose, (b) ribose, and (c) $2^{\prime}$ O-methoxyethyl ribose corresponding to oligos 7, 8, and 9 respectively. The total amount of fragmentation for each oligo has been normalized to 1. Each type of fragmentation includes contributions from all charge states for the fragment.

\section{Comparison of Phosphodiester and Phosphorothioate Backbones}

Since one of the most commonly employed chemical modifications in antisense compounds is the replacement of an oxygen atom of the phosphodiester linkage $\left(\mathrm{PO}_{4}\right)$ with a sulfur atom to form a phosphorothioate linkage $\left(\mathrm{PSO}_{3}\right)$, a comparison between the fragmentation patterns of a 21-mer phosphodiester oligonucleotide (Oligo 1) and a 21-mer phosphorothioate oligonucleotide (Oligo 2) with the same sequence was made (Table 2). In Table 2, the absolute abundances (arbitrary units) for the each charge state of a fragment are summed together and then the fragments are normalized to 1 based on the most abundant fragment. For both the phosphodiester and the phosphorothioate oligonucleotides $\mathrm{a}_{\mathrm{n}}-\mathrm{B}$ and $\mathrm{w}_{\mathrm{n}}$ fragment ions dominate, accounting for over $85 \%$ of the fragment ions. For both oligonucleotides, no $\mathrm{a}_{\mathrm{n}}-\mathrm{B}$ fragment ions and decreased $\mathrm{w}_{\mathrm{n}}$ fragment ions are observed at the $3^{\prime}$ side of $\mathrm{T}$.
Previous studies have also observed a decreased tendency for fragmentation at the $3^{\prime}$ side of a $T[7,8,22$, 26-30]. While McCloskey and coworkers did not observe the $a_{n}-5 \mathrm{MeC}$ fragment ion under CID conditions [12], the $a_{n}-5 \mathrm{MeC}$ fragment ion is observed for both oligonucleotides using the InHex IRMPD fragmentation scheme (Table 2). For both oligonucleotides, $y_{n}$ fragment ions account for $4-6 \%$ of the total fragment ions. In contrast, $b_{n}$ and $c_{n}$ fragment ions each account for less than $4 \%$ of the total fragment ions exclusively in the phosphorothioate oligonucleotide - appreciable abundances of $b_{n}$ and $c_{n}$ fragment ions are not observed in the construct with the phosphodiester backbone. In each case, sufficient fragmentation was observed to confirm the sequence of the oligonucleotides. Since the overall fragmentation patterns are similar for the two different backbones and phosphorothioate backbones are widely used in antisense compounds, all studies presented below employ a full phosphorothioate backbone. 
Table 2. Summed normalized abundances of the different fragment types for Oligo 1 (phosphodiester backbone, PO) and Oligo 2 (phosphorothioate ester backbone, PS)

\begin{tabular}{|c|c|c|c|c|c|c|c|c|c|c|c|c|c|c|}
\hline \multirow[b]{2}{*}{ Sequence } & \multicolumn{2}{|c|}{$a_{n}$} & \multicolumn{2}{|c|}{$a_{n}-B$} & \multicolumn{2}{|c|}{$b_{n}$} & \multicolumn{2}{|c|}{$c_{n}$} & \multicolumn{2}{|c|}{$w_{n}$} & \multicolumn{2}{|c|}{$x_{n}$} & \multicolumn{2}{|c|}{$y_{n}$} \\
\hline & $\mathrm{PO}$ & PS & $\mathrm{PO}$ & PS & $\mathrm{PO}$ & PS & $\mathrm{PO}$ & PS & $\mathrm{PO}$ & PS & $\mathrm{PO}$ & PS & $\mathrm{PO}$ & PS \\
\hline \multicolumn{15}{|l|}{$T$} \\
\hline \multicolumn{15}{|l|}{ G } \\
\hline A & & & 0.43 & 1.00 & & & & & & & & & & \\
\hline 5 & & & 0.05 & 0.17 & & 0.06 & & 0.10 & & & & & & \\
\hline G & & & 0.08 & 0.08 & & 0.01 & & 0.01 & & & & & & \\
\hline G & 0.01 & & 0.16 & 0.21 & & & & & & & & & & \\
\hline A & & & 1.00 & 0.59 & & & & 0.02 & & & & & & \\
\hline $\mathrm{T}$ & & & & & & 0.01 & & & 0.12 & 0.01 & & & 0.004 & \\
\hline G & & & 0.06 & 0.09 & & & & & & & & & & \\
\hline 5 & & 0.10 & 0.03 & & & & & 0.03 & 0.02 & & & & & \\
\hline 5 & & 0.16 & 0.03 & & & & & 0.05 & & & & & & \\
\hline A & & & 0.28 & 0.09 & & & & & 0.20 & 0.01 & & & & \\
\hline G & & & 0.01 & 0.03 & & & & & 0.56 & 0.02 & & & 0.18 & 0.07 \\
\hline 5 & & & 0.01 & & & & & & 0.26 & 0.18 & & & & \\
\hline$T$ & & & & & & & & & 0.30 & 0.09 & & & 0.01 & 0.03 \\
\hline$T$ & & & & & & & & & 0.07 & & & & & \\
\hline G & & & & & & & & & & & & & & 0.03 \\
\hline G & & & & & & & & & 0.13 & 0.35 & & & & 0.08 \\
\hline G & & & & & & & & & 0.23 & 0.49 & & & 0.01 & 0.09 \\
\hline 5 & & & & & & & & & 0.15 & 0.80 & & & & \\
\hline $\mathrm{T}$ & & & & & & & & & & & & & & \\
\hline
\end{tabular}

Note: 5 = 5-Methyl C.

\section{Fragmentation Patterns of Phosphorothioate Oligonucleotides}

Six phosphorothioate deoxyoligonucleotides ranging in size from an 18- to a 21-mer were fragmented using InHex IRMPD (Table 1, oligos 2-7). Sequence information was obtained for 11-13 bases in from both the $5^{\prime}$ and $3^{\prime}$ ends with complementary fragments observed for the 3-5 bases in the middle of the oligonucleotide, resulting in complete sequence information for all six oligonucleotides. A representative InHex IRMPD spectrum is shown in Figure 1A. The spectrum is dominated by $\mathrm{a}_{\mathrm{n}}-\mathrm{B}$ and $\mathrm{w}_{\mathrm{n}}$ fragment ions that provide near complete sequence coverage. Low level $a_{n}, b_{n}, c_{n}, x_{n}$, and $y_{n}$ fragment ions are observed as well. In addition to the primary fragments, lower abundance species corresponding to deadenylation, dehydration, and internal fragmentation are observed.

Fragments that contain the $5^{\prime}$ end of oligonucleotides are dominated by the $\mathrm{a}_{\mathrm{n}}-\mathrm{B}$ fragment ions, which represent $30 \%$ of the total fragment ion abundance. With a few minor exceptions, the fragment ion $a_{n}-T$ is not observed. In cases where the T occurs within 3 bases of the $5^{\prime}$ end or when there are multiple $T^{\prime}$ 's in a row (i.e., TT) within 3 bases of the $5^{\prime}$ end, the $a_{n}$-T fragment ion is observed at low abundance. In addition to $\mathrm{a}_{\mathrm{n}}-\mathrm{B}$ fragment ions, $a_{n}, b_{n}$, and $c_{n}$ fragment ions are observed at lower abundances $(3.2 \%, 8.7 \%, 7.0 \%$, respectively). No $d_{n}$ fragment ions are observed for any of the oligonucleotides studied under InHex IRMPD conditions.
Fragments that contain the $3^{\prime}$ end of the oligonucleotides are dominated by $\mathrm{w}_{\mathrm{n}}$ fragment ions, accounting for $32.1 \%$ of the total fragment ion abundance. The abundance of $\mathrm{w}_{\mathrm{n}}$ fragment ions formed by fragmentation $3^{\prime}$ to a $\mathrm{T}$ are decreased relative to other bases. Also, $x_{n}$ and $y_{n}$ fragment ions account for $3.3 \%$ and $15.7 \%$ of the total fragment ion abundance, respectively. No $z_{n}$ fragment ions were observed for any of the oligonucleotides studied under InHex IRMPD conditions.

For most phosphorothioate deoxyoligonucleotides in the size range studied herein, identification of the $a_{n}-B$ and $\mathrm{w}_{\mathrm{n}}$ fragment ions yields complete sequence information. The most abundant fragment ions correspond to $a_{n}-B$ or $w_{n}$ associated with the loss of an $A$ and fragmentation on the $3^{\prime}$ side of the sugar from which the A was lost. Oligonucleotides that have $5^{\prime}-\mathrm{TN}-3^{\prime}$ (where $\mathrm{N}=\mathrm{A}, \mathrm{G}, \mathrm{C}$, or $\mathrm{T}$ ) as part of the sequence may have gaps in the $a_{n}-B$ and $w_{n}$ series because fragmentation $3^{\prime}$ to a $\mathrm{T}$ is disfavored. For example, if the $\mathrm{a}_{8}-\mathrm{B}$ and $\mathrm{a}_{10}-\mathrm{B}$ fragment ions are observed but the $\mathrm{a}_{9}-\mathrm{B}$ fragment ion is not observed, the mass difference between the $\mathrm{a}_{8}-\mathrm{B}$ and $\mathrm{a}_{10}-\mathrm{B}$ fragment ions will correspond mostly likely to a $\mathrm{T}$ nucleotide and one other nucleotide. Additionally, the T nucleotide will be at the 9th position since no $\mathrm{a}_{9}-\mathrm{B}$ fragment ion was observed. A $5^{\prime}-\mathrm{NT}-3^{\prime}$ (where $\mathrm{N}=\mathrm{A}, \mathrm{G}$, or $\mathrm{C}$ ) as part of the sequence will not cause a gap in the $a_{n}-B$ and $w_{n}$ series since fragmentation will occur on the $3^{\prime}$ side of $\mathrm{N}$. In most cases, the usually weak fragment ions $a_{n}, b_{n}, c_{n}, x_{n}$, and $y_{n}$ can be used to fill in the sequence gaps caused by $5^{\prime}-\mathrm{TN}-3^{\prime}$ sequences. 
Table 3. Comparison of integrated fragment abundance (by type) as a function of 2 ' sugar substitute

\begin{tabular}{|c|c|c|c|c|c|c|c|}
\hline Oligo & $a_{n}$ & $a_{n}-B$ & $b_{n}$ & $c_{n}$ & $w_{n}$ & $x_{n}$ & $y_{n}$ \\
\hline 7 (2' deoxyribose) & $8.0 \%$ & $34.5 \%$ & $2.4 \%$ & $6.1 \%$ & $33.2 \%$ & $4.6 \%$ & $11.2 \%$ \\
\hline 8 (ribose) & $0.2 \%$ & $5.2 \%$ & $13.4 \%$ & $16.2 \%$ & $2.2 \%$ & $21.4 \%$ & $41.4 \%$ \\
\hline 9 (2' MOE) & $0.8 \%$ & $1.6 \%$ & $11.6 \%$ & $23.1 \%$ & $12.3 \%$ & $22.5 \%$ & $28.2 \%$ \\
\hline
\end{tabular}

\section{Fragment Patterns as a Function of 2' Sugar Substituents}

The same sequence was made as a phosphorothioate oligonucleotide with three different substituents at the $2^{\prime}$ position: (1) 2' deoxy (DNA), (2) 2' hydroxyl (RNA), and (3) 2' methoxyethyl (MOE) (Table 1, oligos 7, 8, and 9). The fragmentation pattern changed as a function of the substituent at the $2^{\prime}$ position (Figure 2 , Table 3). All three oligonucleotides show significant fragmentation 10-13 bases from both the $3^{\prime}$ and $5^{\prime}$ ends. Under typical InHex IRMPD conditions (500 ms irradiation times and 13.5 watts laser power), both the DNA and RNA sequence fragment readily and generate abundant fragments ranging 2 to 13 nucleotides in length (Figure 2A and B). In contrast, the MOE sequence requires significantly higher laser power (22 watts) and the resulting spectra are dominated by relatively small $c_{n}$ and $y_{n}$ ions (2 to 5 nucleotides in length) (Figure 2C). Regardless, fragmentation ions yielding full sequence coverage were obtained for all of these oligonucleotides.

The amount of $a_{n}$ and $a_{n}-B$ fragment ions decreased as the $2^{\prime}$ substituent was changed from a $\mathrm{H}$ to $\mathrm{OH}$ to MOE (Table 3). The observed order of preference for $\mathrm{a}_{\mathrm{n}}-\mathrm{B}$ fragment ions for DNA and RNA oligonucleotides is $\mathrm{A}>\mathrm{C}>\mathrm{G}>>\mathrm{T}$. In contrast, the observed order of preference for $a_{n}-B$ fragment ions for a MOE oligo is $\mathrm{C}>\mathrm{G} \approx \mathrm{A}>>\mathrm{T}$. The contribution from both $b_{n}$ and $c_{n}$ fragment ions increases as the $2^{\prime}$ sugar substituent is changed (Table 3 ). Interestingly, the observed order of preference for $b_{n}$ and $c_{n}$ fragment ions is not influenced by the $2^{\prime}$ substituent: $C \approx A>G \approx T$ and $A>C \approx T>G$, respectively.

The abundance of $w_{n}$ and $y_{n}$ fragment ions change with the $2^{\prime}$ substituent on the sugar but not in a systematic fashion (Table 3 ). The observed order of preference for formation of $w_{n}$ and $y_{n}$ fragment ions for DNA and RNA sequences is: $\mathrm{A}>\mathrm{G}>\mathrm{C}>\mathrm{T}$ and $A>C \approx G \approx T$, respectively; while for $M O E$ sequence, it is: $\mathrm{G}>\mathrm{T}>\mathrm{C}>\mathrm{A}$ and $\mathrm{C}>\mathrm{T}>\mathrm{G}>\mathrm{A}$, respectively. The contribution from $x_{n}$ fragment ions increases as the 2 ' sugar substituent is changed (see Table 3 ). The observed order of preference for formation of $x_{n}$ fragment ions for DNA and RNA sequences is $C>T>G>A$. In contrast, the observed preference for $\mathrm{MOE}$ sequence is $\mathrm{T}>\mathrm{G}>\mathrm{C}>\mathrm{A}$. For all three substituents, there is a decreased tendency for fragmentation $3^{\prime}$ to a $\mathrm{T}$ with the exception that an intense $\mathrm{w}_{2}$ fragment ion is observed in the MOE sequence. In general, MOE sequences show significant fragmentation $3^{\prime}$ to a $\mathrm{T}$ within three bases of the $3^{\prime}$ terminus of the oligonucleotide.

\section{Fragmentation Patterns of Chimeric Oligonucleotide}

Chimeric oligonucleotides, in which the MOE moieties are incorporated at the $2^{\prime}$ position of the 4 most distal nucleotides of each end (MOE wings), are termed "gapmers". Gapmers are increasingly used as antisense compounds since they have enhanced affinity and nuclease resistance due to the MOE wings [31]. When there is a mixture of $2^{\prime}$ substituents, fragmentation preferentially occurs at nucleotides with 2' deoxy groups instead of nucleotides that contain 2' methoxyethyl groups. As shown in Figure 3, the fragmentation pattern of a phosphorothioate deoxyoligonucleotide (Oligo 6) was compared to a phosphorothioate gapmer oligonucleotide (Oligo 10) of the same sequence which has MOE wings and deoxy groups in the gap (Table 1). Overall, both Oligo 6 and 10 have similar abundances of the different types of fragment ions: $1.1 \%$ and $0.5 \% \mathrm{a}_{\mathrm{n}}$ fragment ions; $20.6 \%$ and $23 \% \mathrm{a}_{\mathrm{n}}-\mathrm{B}$ fragment ions; $19.6 \%$ and $22.1 \% b_{n}$ fragment ions; $3.9 \%$ and $3.9 \% c_{n}$ fragment ions; $20.8 \%$ and $18.7 \% \mathrm{w}_{\mathrm{n}}$ fragment ions; $1.2 \%$ and $1.7 \% \mathrm{x}_{\mathrm{n}}$ fragment ions; and $32.8 \%$ and $30.1 \% \mathrm{y}_{\mathrm{n}}$ fragment ions, respectively (Figure 3 ). The fragmentation patterns differ significantly only at the nucleotides that have MOE groups in the $2^{\prime}$ position. For example, Oligo 6 shows significant $a_{n}-B$ and $w_{n}$ fragmentation throughout the sequence while Oligo 10 shows significant $a_{n}-B$ and $w_{n}$ fragmentation only at the deoxynucleotides and not at the MOE nucleotides. For Oligo 10, only significant amounts of $b_{n}$ and $y_{n}$ fragment ions are observed at the MOE wings. Identification of the $b_{n}, c_{n}, x_{n}$ and $y_{n}$ fragment ions is necessary to obtain complete sequence information for Oligo 10.

\section{General Fragmentation Patterns}

From this study of fragmentation patterns of various oligonucleotides, the following general observations can be made:

1. For phosphorothioate and phosphodiester oligodeoxynucleotides, the $a_{n}-B$ and $w_{n}$ fragment ions dominate with extensive fragmentation occurring $3^{\prime}$ to A nucleotides. Additionally, for 20 mer oligonucleotides, complementary $a_{n}-B$ and $w_{n}$ fragment ions are observed when the fragments are close in length (e.g., $\mathrm{a}_{11}-\mathrm{B}$ and $\mathrm{w}_{9}$ are observed) but complementary fragments are not observed when the two fragments are differ significantly in length (e. g. $\mathrm{a}_{3}-\mathrm{B}$ is observed but $\mathrm{w}_{17}$ is not observed) 2. For oligonucleotides with hydroxyl or MOE groups, $b_{n}, c_{n}, x_{n}$ and $y_{n}$ fragment ions dominate with extensive fragmentation generally oc- 


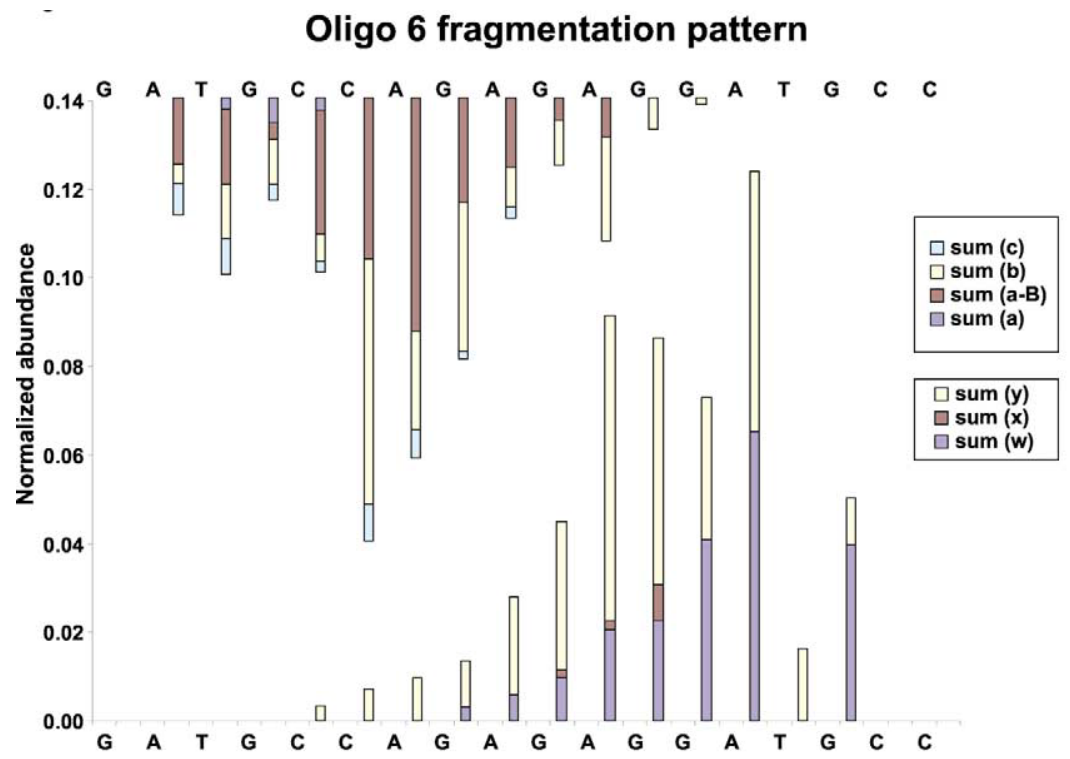

(a)

\section{Oligo 10 fragmentation pattern}

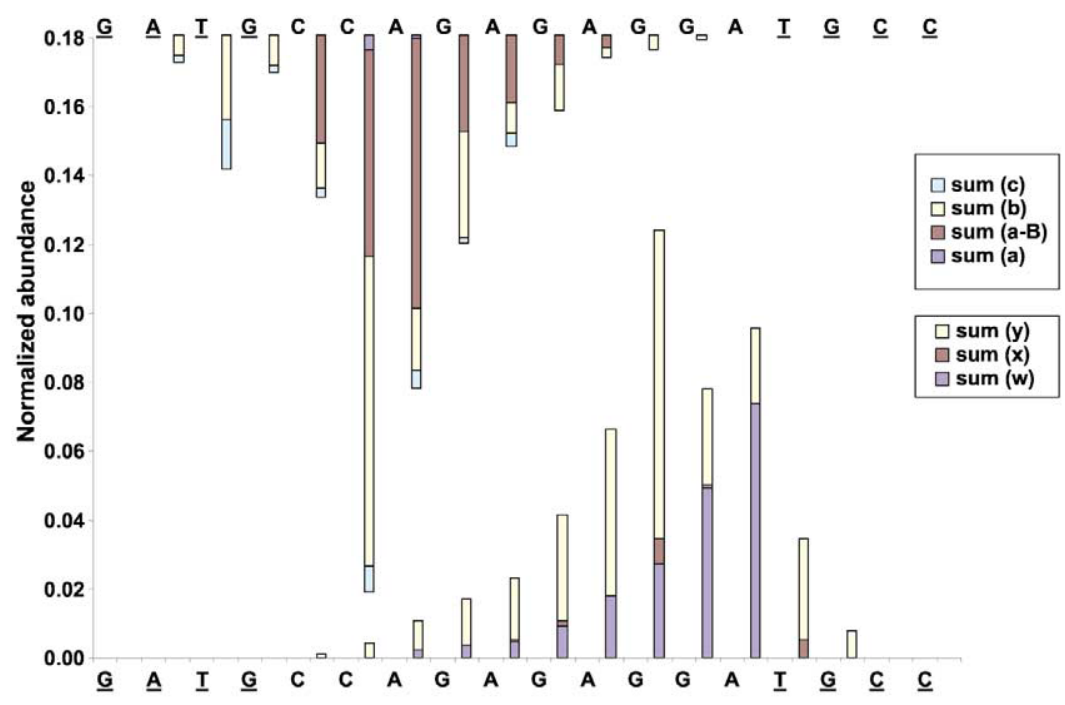

(b)

Figure 3. InHex IRMPD fragmentation patterns for the phosphorothioate oligonucleotide 5'GATG55AGAGAGGATG55-3' in which the 2' substitutions are (a) uniformly deoxyribose (Oligo 6) and (b) deoxyribose with MOE substituents on the 4 distal bases on each end (underlined bases) (Oligo 10). The total amount of fragmentation for each oligonucleotide has been normalized to 1. Each type of fragmentation includes contributions from all charge states for the fragment type.

curring $3^{\prime}$ to $\mathrm{A}$ and $\mathrm{C}$ nucleotides. Similar to the oligodeoxynucleotides, complementary $b_{n}$ and $x_{n}$ fragment ions as well as complementary $c_{n}$ and $y_{n}$ ions are observed near the middle of the sequence but for 20-mer oligonucleotides generally does not extend the full length of the oligonucleotide. In most cases, the complementary $c_{n}$ and $y_{n}$ fragment ions are more intense than the complementary $b_{n}$ and $x_{n}$ fragment ions. 3. For all oligonucleotides studied in this work, decreased fragmentation is observed $3^{\prime}$ to T nucleotides regardless of the type of fragment ions formed. In most cases, no $a_{n}-B$ and $w_{n}$ fragment ions are observed at $T$ nucleotides regardless of the 2 ' substituent of the sugar.

\section{Fragmentation Mechanisms Proposed in the Literature}

Several groups have proposed fragmentation mechanisms to explain the observed $a_{n}-B$ and $w_{n}$ fragment ions. McLuckey and coworkers proposed a 2-step, 1,2 elimination scheme to explain the formation of $a_{n}-B$ and 
$\mathrm{w}_{\mathrm{n}}$ fragment ions where the base can be lost either as a neutral or an anion [32]. They observed a preference for base loss as $\mathrm{A}>\mathrm{G}>\mathrm{T}>\mathrm{C}$. Rodgers et al. proposed a proton bound intermediate to explain the loss of bases as both neutrals and as anions [33]. They observed a base loss preference of $A>T \gg G>C$. Similar to the McLuckey group, Barry and coworkers proposed a base-catalyzed 2-step, 1,2 elimination scheme where the negatively charged oxygen of the 3 'phosphodiester group abstracts a proton from the $2^{\prime}$ position of the sugar resulting in base loss [13]. The observed preference for nucleobase loss and subsequent formation of $a_{n}-B$ fragment ions is $A>T>G \approx C$. Nordhoff et al. proposed a charge remote, base elimination mechanism whereby the base is protonated through an intramolecular proton transfer followed by cleavage at the $5^{\prime}$ and $3^{\prime}$ phosphate [27]. The observed base loss preference was $A \approx G>C>>T$. Gross and coworkers proposed an intramolecular proton transfer from the $5^{\prime}$ phospate to the base resulting in a zwitterionic intermediate from which a neutral base is lost and subsequent cleavage leads to formation of the $a_{n}-B$ and $w_{n}$ ions $[26,28,29]$. Under ESI and source CAD conditions, Gross and coworkers observed that the ease of formation of $a_{n}-B$ fragment ions is $G>C \approx A>>T$. The order of base loss supports their proposed mechanism of protonation of the base by the $5^{\prime}$ phosphate group since the order follows the proton affinity of the bases. It is interesting to note that the preference for formation of $a_{n}-B$ fragment ions is dependent on the fragmentation scheme employed.

All of the above mechanisms rely on the ability of the $3^{\prime}$ phosphate group to interact with the $2^{\prime}$ position of the sugar. The fragmentation patterns of the $2^{\prime}$ substituted oligonucleotides are consistent with the $3^{\prime}$ phosphate group interacting with the $2^{\prime}$ position of the sugar. A significant decrease in $a_{n}-B$ fragment ions is observed when the $2^{\prime}$ substituent is changed from deoxy to hydroxyl to methoxyethyl group (Table 3). In this study, changing from a deoxyoligonucleotide to oligoribonucleotide, the amount of $\mathrm{w}_{\mathrm{n}}$ fragment ions decreases. Interestingly, changing from an oligoribonucleotide to a MOE oligonucleotide causes the formation of $\mathrm{w}_{\mathrm{n}}$ fragment ions to increase. It is possible that an alternative fragmentation pathway for formation of $w_{n}$ fragment ions exists when the $2^{\prime}$ substituent is a methoxyethyl group that is not available when the $2^{\prime}$ substituent is a hydroxyl group. Both the hydroxyl group and the methoxyethoxyl group are electron withdrawing, which stabilizes the N-gylcosidic bond and makes protonation of the nucleobase unfavorable [3437]. This in turn makes loss of a base, which is required for formation of the $a_{n}-B$ fragment ion unfavorable. Additionally, both the hydroxyl and the methoxyethyl groups may sterically hinder the attack of the $3^{\prime}$ phosphate group at the $2^{\prime}$ position of the sugar.

Changing the 2' substituent of the sugar causes other fragmentation pathways to become accessible and/or dominant over the pathways that form $a_{n}-B$ and $w_{n}$ fragment ions. Recently, Leumann and coworkers have proposed two pathways for formation of $c_{n}$ and $y_{n}$ fragment ions in the fragmentation of oligoribonucleotides [38]. One pathway involves the abstraction of the 2 '-hydroxyl proton by the 5 '-phosphate oxygen followed by dissociation of the $5^{\prime}-\mathrm{P}-1 \mathrm{O}$ bond. The second pathway involves transfer of the acidic phosphate proton to the $5^{\prime}$-oxygen followed by cleavage at the $5^{\prime}-\mathrm{P}-\mathrm{O}$ bond. Both pathways for formation of $c_{n}$ and $y_{n}$ fragment ions may be accessible for oligoribonucleotides while only the second pathway is most likely accessible for MOE oligonucleotides, consistent with the observation that $\mathrm{y}_{\mathrm{n}}$ fragment ions are more abundant in oligoribonucleotides than in MOE oligonucleotides (Table 3).

\section{Conclusions}

When oligodeoxynucleotides are fragmented by InHex IRMPD, it is primarily the $a_{n}-B$ and $w_{n}$ fragment ions that are most useful for sequence confirmation, regardless of whether the backbone is comprised of phosphodiester or phosphorothioate linkages. We have shown that $b_{n}, c_{n}, x_{n}$ and $y_{n}$ fragment ions provides significant sequence information and are critical for sequence confirmation of $2^{\prime}$ modified oligonucleotides. Although modifications at the $2^{\prime}$ position of the sugar render them more refractory to fragmentation, increasing the laser power used for InHex IRMPD causes fragmentation sufficient for sequence confirmation of these oligonucleotides. In cases where there is a mixture of 2'substituents, fragmentation predominately occurs at the $2^{\prime}$ deoxy groups. Regardless of the 2' substituent, fragmentation $3^{\prime}$ to a $\mathrm{T}$ is significantly decreased or absent and may cause gaps in the sequence. However, gaps in the sequence corresponding to a $\mathrm{T}$ nucleotide plus an $\mathrm{N}$ nucleotide will have the sequence order $5^{\prime}-\mathrm{TN}-3^{\prime}$ and not $5^{\prime}-\mathrm{NT}-3^{\prime}$. Thus, gaps in the sequence caused by lack of fragmentation do not prevent confirmation of the sequence. Based on the observations in this study, the InHex IRMPD fragmentation scheme provides a rapid and accurate means of sequence confirmation for modified oligonucleotides.

\section{References}

1. Cook, P. D. Medicinal Chemistry Strategies for Antisense Research. Antisense Research and Applications; In Crooke, S. T.; Lebleu, B., Eds.; CRC Press: Boca Raton, 1993; 149-187.

2. Fenn, J. B.; Mann, M.; Meng, C. K.; Wong, S. F.; Whitehouse, C. M. Electrospray Ionization for Mass Spectrometry of Large Biomolecules. Science 1989, 246, 64-71.

3. Smith, R. D.; Loo, J. A.; Edmonds, C. G.; Barinaga, C. J.; Udseth, H. R. New Developments in Biochemical Mass Spectrometry: Electrospray Ionization. Anal. Chem. 1990, 62(9), 882-899.

4. Smith, R. D.; Loo, J. A.; Ogorzalek-Loo, R. R.; Busman, M.; Udseth, H. R. Principles and Practice of Electrospray Ionization Mass-Spectrometry for Large Polypeptides and Proteins. Mass Spectrom. Rev. 1991, 10(5), 359-451. 
5. Karas, M.; Bachmann, D.; Bahr, U.; Hillenkamp, F. MatrixAssisted Ultraviolet Laser Desorption of Non-Volatile Compounds. Int. J. Mass Spectrom. Ion Processes 1987, 78, 53-68.

6. Bahr, U.; Karas, M.; Hillenkamp, F. Analysis of Biopolymers by Matrix-Assisted LaserDesorption/Ionization(MALDI)MassSpectrometry. Fresenius J. Anal. Chem. 1994, 348(12), 783-791.

7. Little, D. P.; Aaserud, D. J.; Valaskovic, G. A.; McLafferty, F. W. Sequence Information from 42-108-mer DNAs (Complete for a 50-mer) by Tandem Mass Spectrometry. J. Am. Chem. Soc. 1996, 118(39), 9352-9359.

8. Little, D. P.; McLafferty, F. W. Sequencing 50-mer DNAs Using Electrospray Tandem Mass Spectrometry and Complementary Fragmentation Methods. J. Am. Chem. Soc. 1995, 117, 6783-6784.

9. Little, D. P.; Thannhauser, T. W.; McLafferty, F. W. Verification of 50- to 100-mer DNA and RNA Sequences with HighResolution Mass Spectrometry. Proc. Natl. Acad. Sci. U.S.A. 1995, 92, 2318-2322.

10. McLuckey, S. A.; Habibigoudarzi, S. Ion Trap-Tandem Mass Spectrometry Applied to Small Multiply Charged Oligonucleotides with a Modified Base. J. Am. Soc. Mass Spectrom. 1994, 5(8), 740-747.

11. Ni, J. Pomerantz, S. C. McCloskey, J. A. Rapid Sequencing of Modified Oligonucleotides Using Tandem Mass Sectrometry with Electrospray Ionization. Nucleic Acids Symposium Series. Proceedings of the 23rd Symposium on Nucleic Acids Chemistry; 1996; pp 113-114.

12. Ni, J.; Pomerantz, S. C.; Rozenski, J.; Zhang, Y.; McCloskey, J. A. Interpretation of Oligonucleotide Mass Spectra for Determination of Sequence Using Electrospray Ionization and Tandem Mass Spectrometry. Anal. Chem. 1996, 68(13), 1989-1999.

13. Barry, J. P.; Vouros, P.; Vanschepdael, A.; Law, S. J. Mass and Sequence Verification of Modified Oligonucleotides Using Electrospray Tandem Mass Spectrometry. J. Mass Spectrom. 1995, 30(7), 993-1006.

14. Hofstadler, S. A.; Sannes-Lowery, K. A.; Griffey, R. H. Infrared Multiphoton Dissociation in an External Ion Reservoir. Anal. Chem. 1999, 71, 2067-2070.

15. Anderson, G. A.; Bruce, J. E. ICR $2 L S$ version 2.18. Pacific Northwest National Laboratory: Richland, WA, 1996.

16. McLuckey, S. A.; Van Berkel, G. J.; Glish, G. L. Tandem Mass Spectrometry of Small, Multiply Charged Oligonucleotides. J. Am. Soc. Mass Spectrom. 1992, 3(1), 60-70.

17. Little, D. P.; Chorush, R. A.; Speir, J. P.; Senko, M. W.; Kelleher, N. L.; McLafferty, F. W. Rapid Sequencing of Oligonucleotides by High-Resolution Mass Spectrometry. J. Am. Chem. Soc. 1994, 116(11), 4893-4897.

18. Little, D. P.; Speir, J. P.; Senko, M. W.; O'Connor, P. B.; McLafferty, F. W. Infrared Multiphoton Dissociation of Large Multiply Charged Ions for Biomolecule Sequencing. Anal. Chem. 1994, 66(18), 2809-15.

19. Aaserud, D. J.; Guan, Z.; Little, D. P.; McLafferty, F. W. DNA Sequencing with Blackbody Infrared Radiative Dissociation of Electrosprayed Ions. Int. J. Mass Spectrom. Ion Processes 1997, 167(168), 705-712.

20. Sannes-Lowery, K. A.; Hofstadler, S. A. Characterization of Multipole Storage Assisted Dissociation: Implications for Electrospray Ionization Mass Spectrometry Characterization of Biomolecules. J. Am. Soc. Mass Spectrom. 2000, 11(1), 1-9.

21. Tang, W.; Zhu, L.; Smith, L. M. Controlling DNA Fragmentation in MALDI-MS by Chemical Modification. Anal. Chem. 1997, 69(3), 302-312.

22. Wang, Y.; Taylor, J.-S.; Gross, M. L. Differentiation of Isomeric Photomodified Oligodeoxynucleotides by Fragmentation of Ions Produced by Matrix-Assisted Laser Desorption Ionization and Electrospray Ionization. J. Am. Soc. Mass Spectrom. 1999, 10(4), 329-338.
23. Hannis, J. C.; Muddiman, D. C. Tailoring the gas-phase dissociation and determining the relative energy of activation for dissociation of 7-deaza purine modified oligonucleotides containing a repeating motif. Int. J. of Mass Spectrom. 2002, 219(1), 139-150.

24. Wang, Y.; Men, L.; Vivekananda, S. Fragmentation of Deprotonated Ions of Oligodeoxynucleotides Carrying a 5-Formyluracil or 2-Aminoimidazolone. J. Am. Soc. Mass Spectrom. 2002, 13, 1190-1194.

25. Bartlett, M. G.; McCloskey, J. A.; Manalili, S.; Griffey, R. H. The Effect of Backbone Charge on the Collision-Induced Dissociation of Oligonucleotides. J. Mass Spectrom. 1996, 31(11), 12771283.

26. Wan, K. X.; Gross, M. L. Fragmentation Mechanisms of Oligodeoxynucleotides: Effects of Replacing Phosphates with Methylphosphonates and Thymines with Other Bases in T-Rich Sequences. J. Am. Soc. Mass Spectrom. 2001, 12(5), 580-589.

27. Nordhoff, E.; Karas, M.; Cramer, R.; Hahner, S.; Hillenkamp, F.; Kirpekar, F.; Lezius, A.; Muth, J.; Meier, C.; Engels, J. W. Direct Mass Spectrometric Sequencing of Low-Picomole Amounts of Oligodeoxynucleotides with up to 21 Bases by Matrix-Assisted Laser Desorption Ionization Mass Spectrometry. J. Mass Spectrom. 1995, 30(1), 99-112.

28. Wang, Z.; Wan, K. X.; Ramanathan, R.; Taylor, J. S.; Gross, M. L. Structure and Fragmentation Mechanisms of Isomeric T-Rich Oligodeoxynucleotides: A Comparison of Four Tandem Mass Spectrometric Methods. J. Am. Soc. Mass Spectrom. 1998, 9(7), 683-691.

29. Wan, K. X.; Gross, J.; Hillenkamp, F.; Gross, M. L. Fragmentation Mechanisms of Oligodeoxynucleotides Studied by H/D Exchange and Electrospray Ionization Tandem Mass Spectrometry. J. Am. Soc. Mass Spectrom. 2001, 12(2), 193-205.

30. Gross, J.; Hillenkamp, F.; Wan, K. X.; Gross, M. L. Metastable Decay of Negatively Charged Oligodeoxynucleotides Analyzed with Ultraviolet Matrix-Assisted Laser Desorption/ Ionization Post-Source Decay and Deuterium Exchange. J. Am. Soc. Mass Spectrom. 2001, 12(2), 180-192.

31. Altmann, K.-H.; Dean, N. M.; Fabbro, D.; Freier, S. M.; Geiger, T.; Haener, R.; Huesken, D.; Martin, P.; Monia, B. P. Second Generation of Antisense Oligonucleotides. From Nuclease Resistance to Biological Efficacy in Animals. Chimia 1996, 4, $168-176$.

32. McLuckey, S. A.; Habibi-Goudarzi, S. Decompositions of Multiply Charged Oligonucleotide Anions. J. Am. Chem. Soc. 1993, 115, 12085-12095.

33. Rodgers, M. T.; Campbell, S.; Marzluff, E. M.; Beauchamp, J. L. Low-Energy Collision-Induced Dissociation of Deprotonated Dinucleotides: Determination of the Energetically Favored Dissociation Pathways and the Relative Acidities of the Nucleic Acid Bases. Int. J. Mass Spectrom. Ion Processes 1994, 137, 121-149.

34. Garrett, E. R.; Seydel, J. K.; Sharpen, A. J. The Acid-Catalyzed Solvolysis of Pyrimidine Nucleosides. J. Org. Chem. 1966, 31(7), 2219-2227.

35. Garrett, E. R.; Mehta, P.J. Solvolysis of Adenine Nucleosides. II. Effects of Sugars and Adenine Substituents on Alkaline Solvolyses. J. Am. Chem. Soc. 1972, 94(24), 8542-8547.

36. Garrett, E. R.; Mehta, P. J. Solvolysis of Adenine Nucleosides. I. Effects of Sugars and Adenine Substituents on Acid Solvolyses. J. Am. Chem. Soc. 1972, 94(24), 8532-8541.

37. York, J. L. Effect of the Structure of the Glycon on the Acid-Catalyzed Hydrolysis of Adenine Nucleosides. J. Org. Chem. 1981, 46(10), 2171-2173.

38. Schurch, S.; Bernal-Mendez, E.; Leumann, C. J. Electrospray Tandem Mass Spectrometry of Mixed-Sequence RNA/DNA Oligonucleotides. J. Am. Soc. Mass Spectrom. 2002, 13(8), $936-$ 945. 